\title{
Lessons learnt from a case of missed central hypothyroidism
}

\author{
TessaGlyn', Beverley Harris ${ }^{2}$ and Kate Allen ${ }^{1}$ \\ 1Diabetes and Endocrinology and ${ }^{2} \mathrm{Clinical}$ Biochemist, Royal United Hospital, Bath, UK
}

\author{
Correspondence \\ should be addressed \\ to T Glyn \\ Email \\ tessa.glyn@nhs.net
}

\section{Summary}

We present the case of a 57-year-old lady who had a delayed diagnosis of central hypothyroidism on a background of Grave's thyrotoxicosis and a partial thyroidectomy. During the twenty years following her partial thyroidectomy, the patient developed a constellation of symptoms and new diagnoses, which were investigated by numerous specialists from various fields, namely rheumatology, renal and respiratory. She developed significantly impaired renal function and raised creatine kinase (CK). She was also referred to a tertiary neurology service for investigation of myositis, which resulted in inconclusive muscle biopsies. Recurrently normal TSH results reassured clinicians that this did not relate to previous thyroid dysfunction. In 2015, she developed increased shortness of breath and was found to have a significant pericardial effusion. The clinical biochemist reviewed this lady's blood results and elected to add on a free T4 (fT4) and free T3 (fT3), which were found to be $<0.4 \mathrm{pmol} / \mathrm{L}$ (normal range (NR): $12-22 \mathrm{pmol} / \mathrm{L}$ ) and $0.3 \mathrm{pmol} / \mathrm{L}$ (NR: $3.1-6.8 \mathrm{pmol} / \mathrm{L}$ ), respectively. She was referred urgently to the endocrine services and commenced on Levothyroxine replacement for profound central hypothyroidism. Her other pituitary hormones and MRI were normal. In the following year, her eGFR and CK normalised, and her myositis symptoms, breathlessness and pericardial effusion resolved. One year following initiation of Levothyroxine, her fT4 and fT3 were in the normal range for the first time. This case highlights the pitfalls of relying purely on TSH for excluding hypothyroidism and the devastating effect the delay in diagnosis had upon this patient.

\section{Learning points:}

- Isolated central hypothyroidism is very rare, but should be considered irrespective of previous thyroid disorders.

- If clinicians have a strong suspicion that a patient may have hypothyroidism despite normal TSH, they should ensure they measure fT3 and fT4.

- Laboratories that do not perform fT3 and fT4 routinely should review advice sent to requesting clinicians to include a statement explaining that a normal TSH excludes primary but not secondary hypothyroidism.

- Thyroid function tests should be performed routinely in patients presenting with renal impairment or a raised CK.

\section{Background}

Central hypothyroidisim is rare with an incidence of 1 in 80 000-120 000 individuals (1). It is characterised by insufficient thyroid gland stimulation by TSH, resulting from hypothalamic or pituitary dysfunction. It is rarely isolated and occurs more commonly in conjunction with other pituitary hormone deficiencies, as well as neurological symptoms and signs resulting from the causative lesion. Symptoms tend to be similar to primary thyroid dysfunction, but according to the literature may be milder (2).

In adults, the commonest causes are pituitary macroadenomas, pituitary surgery or irradiation (3), but there are increasing examples of genetic causes causing isolated central thyroid dysfunction.

With the use of serum TSH as an initial screening test for thyroid dysfunction, the diagnosis of central 
hypothyroidism may be delayed or even missed because most of these patients have normal or low TSH. Hence, many guidelines, including the British Thyroid Association, suggest that if laboratories are unable to identify those specimens that specifically require the measurement of both serum TSH and fT4, then it would be prudent to measure serum TSH and fT4 on all specimens rather than embark on a first-line serum TSH strategy'. They suggest that 'Measurement of serum TSH alone is appropriate after the first investigation in the sequential follow-up of individuals who have not been treated for thyroid disorders and who may be at risk of developing thyroid dysfunction' (4).

Reporting of cases like this is important in order to raise awareness of this condition to general practitioners and other physicians who may be falsely reassured by a normal TSH result. As a result of this case, our biochemistry department has changed its advice to GPs about what to do in the case of a normal TSH : 'TSH within limits excludes primary (but not secondary) hypothyroidism. If there is high clinical suspicion of hypothyroidism, please contact laboratory clinical staff to discuss further tests'.

\section{Case presentation}

The patient initially presented aged 26 in 1988 with an irregular menstrual cycle and was diagnosed during investigations for infertility with Grave's thyrotoxicosis. Following a number of miscarriages, she was commenced on anti-thyroid medication and proceeded to have a successful pregnancy in 1990. She had no specific past medical history, but had a strong family history of autoimmune disorders. She was initially managed medically with carbimazole, but then changed to propylthiouracil because of joint pains. In October 1991, the decision was made to proceed to subtotal thyroidectomy on the grounds that a further pregnancy was not advisable whilst on anti-thyroid medication. Surgery was reported as uneventful, other than a superficial wound infection. At her first post-op appointment, she felt well. Her TSH, alongside her fT3 and T4, were normal. She went through an uncomplicated pregnancy the following year and delivered a healthy baby boy.

Over the next few years, the patient complained of increasing tiredness, aching muscles and joints, with evidence of a proximal myopathy, predominantly affecting her upper arms. Her TSH was checked on multiple occasions and was consistently 2.0-2.5 IU/L.

By 2000, her joint pains had progressed and she developed impaired renal function. She was reviewed extensively by the rheumatologists in regard to her myopathy and arthropathy. She was found to have an erosive osteoarthritis, and her symptoms were managed with steroids and anti-inflammatory analgesia. Alongside these symptoms, her CK rose to above 1000 IU/L (normal range (NR): 25-200 IU/L), and a tertiary referral for suspected myositis was made. Upon review, she was also noted to have bilateral ptosis. Muscle biopsies were undertaken but were inconclusive. The aetiology was thought more likely to be autoimmune, rather than mitochondrial in origin, and further extensive tests were undertaken.

In the forthcoming years, she was referred to the renal department in light of progressive renal impairment. No clear cause for this was found. She was also reviewed by the respiratory department in light of persistent cough and wheeze. In light of the eosinophilia of $0.96 \times 10^{9} / \mathrm{L}$, a high-resolution computed tomography scan and bronchial biopsy were undertaken which showed oedema and some granulomatous change. A tentative diagnosis of Churg-Strauss syndrome was made, despite a negative ANCA. Methotrexate was trialled, but then stopped due to concerns about pulmonary TB on bronchoscopy samples. TB was later excluded. The respiratory physicians ultimately concluded that the most likely diagnosis was asthma.

During work-up for a further muscle biopsy, she was found to have a significant $(2 \mathrm{~cm})$ pericardial effusion. She was referred to Cardiology, who felt that the pericardial effusion was chronic and not compromising, but the aetiology was unclear. A cardiac MRI was arranged to better characterise it. At this point, she was also noted to have a total cholesterol of $12.5 \mathrm{mmol} / \mathrm{L}$, with an LDL cholesterol of $9.7 \mathrm{mmol} / \mathrm{L}$.

Around this time in October 2015, the clinical biochemist reviewed the patient's results and elected to add on an fT4 to her thyroid function tests. TSH was $1.3 \mathrm{IU} / \mathrm{L}$ (NR: 0.3-5 IU/L), fT4: <0.3 pmol/L (NR: 12-22 pmol/L) and fT3: 0.4 pmol/L (NR: 3.1-6.8 pmol/L). A repeat sample was sent and yielded identical results. There was no evidence of interference with the assay. She was urgently referred to an Endocrinology Clinic and a diagnosis of profound central hypothyroidism was made. Her additional symptoms included weight gain, 'puffiness' of the face, constipation and low mood. The remainder of her pituitary function was completed. This revealed a normal 9:00 h cortisol of $582 \mathrm{nmol} / \mathrm{L}(\mathrm{NR}:>$ $300 \mathrm{nmol} / \mathrm{L}$ ), prolactin of $29 \mathrm{IU} / \mathrm{L}$ (NR: 102-496IU/L) and a low IGF-1 of $3.2 \mathrm{nmol} / \mathrm{L}$ (NR: $6.2-24.6 \mathrm{nmol} / \mathrm{L}$ ) suggesting that this axis may also be affected, and raised 


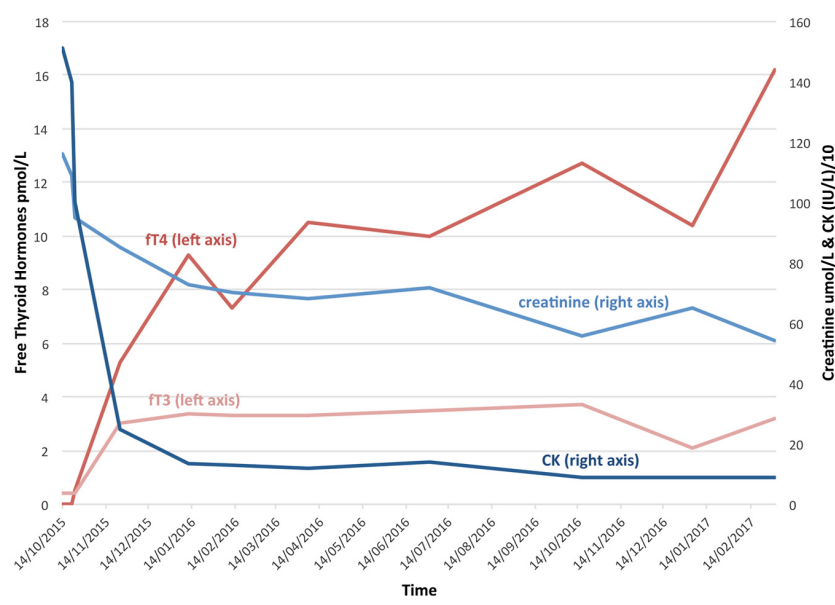

Figure 1

Change in creatinine and CK as free thyroid hormones normalise.

FSH and LH which were perhaps slightly lower than expected for post-menopausal values. Pituitary MRI was normal. It was therefore felt that the predominant axis affected was the hypothalamic-pituitary-thyroid one; however, further evaluation of the growth hormone reserve was to be evaluated once thyroid hormones had been normalised.

She was commenced on Levothyroxine $25 \mu \mathrm{g}$ and the dose was very slowly increased over the forthcoming

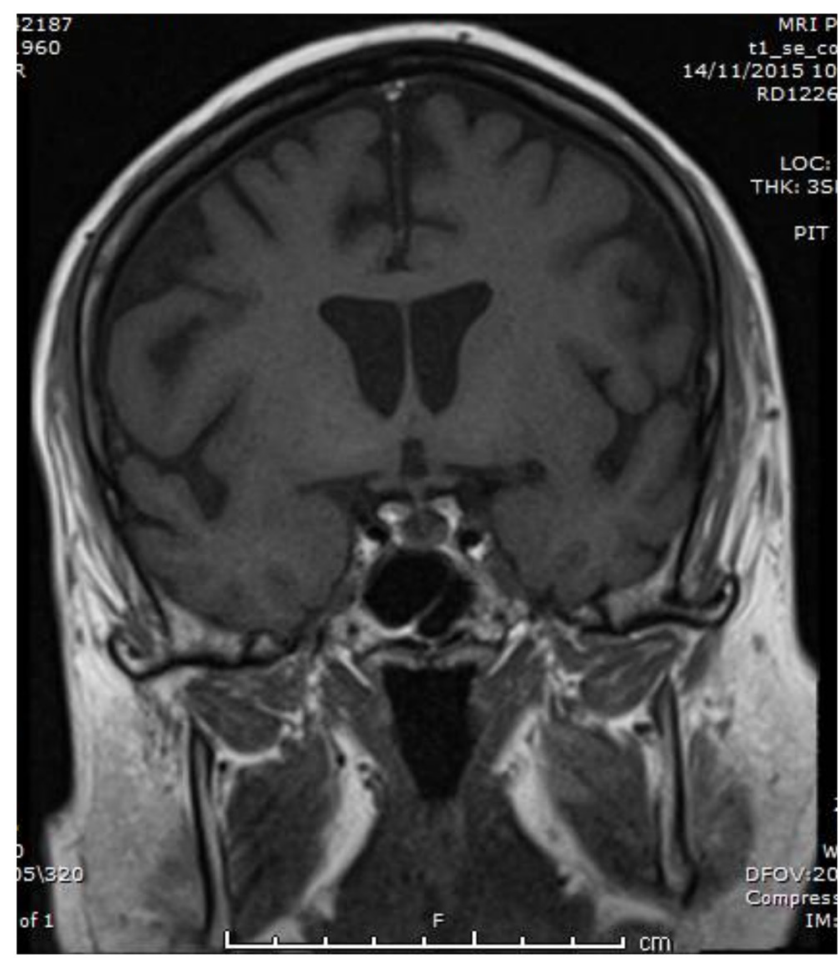

Figure 2

Coronal view of T1 weighted MRI pituitary November 2015.
Table 1 Table of thyroid function test results from 1989 to 2017.

\begin{tabular}{|c|c|c|c|c|}
\hline Date & $\begin{array}{c}\text { TSH } \\
(0.3-5.5 \mathrm{IU} / \mathrm{L})\end{array}$ & $\begin{array}{c}\text { T4 } \\
(54- \\
142 \mathrm{nmol} / \mathrm{L})\end{array}$ & $\begin{array}{c}\text { fT4 } \\
(12-22 \mathrm{pmol} / \mathrm{L})\end{array}$ & $\begin{array}{c}\mathbf{f T 3} \\
(3.1-68 \mathrm{pmol} / \mathrm{L})\end{array}$ \\
\hline Dec 1989 & 0.82 & 168 & & - \\
\hline Jan 1990 & 0.08 & 119 & & - \\
\hline June 1990 & 0.10 & 125 & & 4.32 \\
\hline July 1991 & $<0.13$ & 135 & & 8.75 \\
\hline Dec 1991 & 0.32 & 85 & & 4.77 \\
\hline June 1992 & 0.80 & & - & - \\
\hline Nov 1993 & Euthyroid & & - & - \\
\hline Oct 2006 & 1.44 & & - & - \\
\hline Jan 2007 & 1.64 & & - & - \\
\hline April 2012 & 1.49 & & - & - \\
\hline July 2013 & 1.91 & & - & - \\
\hline Oct 2015 & 1.55 & & $<0.03$ & 0.4 \\
\hline Oct 2016 & 0.15 & & 12.7 & 3.7 \\
\hline Jan 2017 & 0.46 & & 10.4 & 2.1 \\
\hline March 2017 & 0.17 & & 16.2 & 3.2 \\
\hline
\end{tabular}

months, due to the risk of precipitating cardiac arrhythmias. In addition, she felt symptoms of flushing when her dose was increased too quickly. Her eGFR rose quickly from 45 to $73 \mathrm{~mL} / \mathrm{min} / 1.73 \mathrm{~m}^{2}$; CK and myositis symptoms normalised; breathlessness and pericardial effusion resolved. The raised cholesterol also improved and will continue to be monitored. Her symptoms related to hand arthritis persisted, but the rheumatology team felt that this erosive osteoarthritis was likely to be a separate issue to the hypothyroidism.

A year on from diagnosis, her fT4 and fT3 are in the normal range for the first time (fT4: $12.7 \mathrm{pmol} / \mathrm{L}$, fT3: $3.7 \mathrm{pmol} / \mathrm{L}$ ) with a dose of $125 \mu \mathrm{g}$ Levothyroxine. Cautious increase of her Levothyroxine to $150 \mu \mathrm{g}$ has been trialled with the aim of getting her fT 4 in the upper half of the normal range. Her eGFR has normalised to $>90 \mathrm{~mL} / \mathrm{min} / 1.73 \mathrm{~m}^{2}$. Overall, she is feeling vastly better, and will remain under close endocrinology follow-up.

\section{Investigation}

Details are presented in Figs 1 and 2 and Tables 1 and 2 .

Table 2 Pituitary hormone panel at diagnosis.

\begin{tabular}{|c|c|c|}
\hline & Oct 2015 & NRs \\
\hline IGF-1 & $3.2 \mathrm{nmol} / \mathrm{L}$ & $6.2-24.6 \mathrm{nmol} / \mathrm{L}$ \\
\hline $\mathrm{LH}$ & 10.7IU/L & \\
\hline $\mathrm{FSH}$ & $38.7 \mathrm{IU} / \mathrm{L}$ & \\
\hline Oestradiol & $<44 \mathrm{pmol} / \mathrm{L}$ & \\
\hline 09:00 h cortisol & $512 \mathrm{nmol} / \mathrm{L}$ & $>300 \mathrm{nmol} / \mathrm{L}$ \\
\hline Prolactin & $291 \mathrm{IU} / \mathrm{L}$ & 102-496IU/L \\
\hline
\end{tabular}




\section{Treatment}

Management required the introduction of Levothyroxine, but due to the profound nature of her hypothyroidism, up-titration was very slow. There was a concern that rapid correction could induce arrhythmias, cardiac ischaemia or LV dysfunction (5), and also because she complained of feeling hot and flushed when the dose was increased too quickly. The aim is to get her fT4 in the upper half of the normal range.

\section{Outcome and follow-up}

The patient has had 2-monthly reviews with thyroid function tests, in the Endocrinology Clinic since diagnosis 1 year ago. One year on from diagnosis, her fT4 and fT3 are in the normal range.

Our clinical biochemistry team have reviewed their system for thyroid function testing. The cost of performing an fT4 assay on all samples coming to the lab is deemed too prohibitive to run; therefore initially, only a TSH is analysed unless specifically requested. In light of this case, they have changed their advice to GPs and other physicians about what to do in the event of a normal TSH result but high clinical suspicion of hypothyroidism. 'TSH within limits excludes primary (but not secondary) hypothyroidism. If there is high clinical suspicion of hypothyroidism, please contact laboratory clinical staff to discuss further tests'.

\section{Discussion}

This case highlights the possible pitfalls of a system in which thyroid dysfunction is screened purely by testing TSH levels. Practices across the UK vary in regard to first-line screening for thyroid dysfunction (6), which results in disparity and allows for a situation in which cases like this can occur. A case series by Waise and Belchetz describes 6 examples of unsuspected central hypothyroidism, which would not have picked up through isolated TSH testing (7). As suggested by the BTA, it would be beneficial to have a system where initial screening consisted of a full thyroid hormone profile, and then thereafter this could be switched to isolated TSH monitoring if primary hypothyroidism was confirmed (4). At a time of limited resources, this may not be possible, but needs to be carefully considered by each department. If not introduced, other measures such as clear advice on TSH reporting should be put in place to try and limit further cases of missed central hypothyroidism. Cases such as this show how devastating this missed diagnosis can be for the individual involved. It is also worth considering how costly the delay in diagnosis was to the NHS in regard to multiple unnecessary investigations and appointments with other specialties.

This case clearly illustrates the consequences that profound hypothyroidism can have on a variety of body systems, specifically renal dysfunction, myositis and a pericardial effusion. It is therefore of utmost importance that GPs, alongside specialist non-endocrine physicians, are aware of the potential effects of primary or central hypothyroidism, when investigating the underlying cause for such conditions. Once central hypothyroidism is diagnosed, a thorough work-up for evidence of other pituitary dysfunction should be undertaken. Specifically concomitant central adrenal insufficiency should be excluded prior to commencing Levothyroxine replacement in order to avoid precipitating an adrenal crisis (1). Levothyroxine should be introduced at a low daily dose, and built up slowly, whilst monitoring symptoms and fT4 levels (1). JR's case was a clear illustration of how restoration of euthyroidism led to resolution of numerous symptoms and biochemical abnormalities.

Declaration of interest

The authors declare that there is no conflict of interest that could be perceived as prejudicing the impartiality of the research reported.

\section{Funding}

This research did not receive any specific grant from any funding agency in the public, commercial or not-for-profit sector

\section{Patient consent}

Written informed consent has been obtained from the patient for publication of the submitted article and accompanying images.

\section{Patient's perspective}

I feel I have been the victim of too many specialists working in isolation and seeking over-complicated answers and diagnoses - with of course the best of intentions. I feel extremely frustrated that time and again specialists and GPs overlooked the obvious explanation and did not carry out fT3 and fT4 blood tests. 
I feel the quality of my life has been severely impacted - so many investigations at great expense and with potentially negative impact (X-rays, etc.), multiple chronic health conditions and persistent low mood. I feel my life could have been very different if I had been correctly diagnosed at the outset!

I am now on $150 \mu \mathrm{g}$ Levothyroxine. I have more energy, my face shape is changing and ptosis is not an issue. I am always extremely warm and have found managing my temperature difficult. But I do feel in better health. On my first visit to the Endocrinology Department, I had to pause every few minutes whilst walking. I used to fall asleep in the chair all the time, had lots of back and muscle pains and was very constipated. All these things are beginning to change.

My life would have been different if my underactive thyroid had been diagnosed in a timely manner. I will forever be grateful to the clinical biochemist who spotted my abnormal results.

\section{Author contribution statement}

Kate Allen is the named Physician responsible for the patient. Beverley Harris is the Consultant Clinical Biochemist involved with the diagnosis and management of this case. Both have read this report and are happy with the content.

\section{References}

1 Lania A, Persani L \& Beck-Peccoz P 2008 Central hypothyroidism. Pituitary 11 181. (https://doi.org/10.1007/s11102-008-0122-6)

2 Gupta V \& Lee M 2011 Central hypothyroidism. Indian Journal of Endocrinology and Metabolism 15 S99-S106. (https://doi. org/10.4103/2230-8210.83337)

3 Rose SR 2001 Cranial irradiation and central hypothyroidism. Trends in Endocrinology and Metabolism 12 97-104. (https://doi.org/10.1016/ S1043-2760(00)00359-3)

4 British Thyroid Association 2006 UK Guidelines for the Use of Thyroid Function Tests. The Association of Clinical Biochemistry, British Thyroid Association and British Thyroid Foundation. (available at: http://www. british-thyroid-association.org/sandbox/bta2016/uk_guidelines_for_the use_of_thyroid_function_tests.pdf). Accessed on 12 March 2017.

5 Chakera AJ, Pearce SH \& Vaidya B 2012 Treatment for primary hypothyroidism: current approaches and future possibilities. Drug Design, Development and Therapy 6 1-11. (https://doi.org/10.2147/DDDT.S12894)

6 Barth JH, Seth J, Howlett TA \& Freedman DB 1995 A survey of endocrine function testing by clinical biochemistry laboratories in the UK. Annals of Clinical Biochemistry 32 442-449. (https://doi. org/10.1177/000456329503200502)

7 Waise A \& Belchetz PE 2000 Unsuspected central hypothyroidism. BMJ 321 1275-1277. (https://doi.org/10.1136/bmj.321.7271.1275)

Received in final form 10 October 2017

Accepted 15 November 2017 